Correspondence

Kenji Ogawa

ogawak@toumei.hosp.go.jp

Received 8 May 2009

Revised 14 October 2009

Accepted 21 October 2009

\section{Molecular typing of Mycobacterium intracellulare using multilocus variable-number of tandem-repeat analysis: identification of loci and analysis of clinical isolates}

\author{
Kazuya Ichikawa, ${ }^{1}$ Tetsuya Yagi, ${ }^{3}$ Takayuki Inagaki, ${ }^{1,4}$ \\ Makoto Moriyama, ${ }^{1,4,5}$ Taku Nakagawa, ${ }^{1,2}$ Kei-ichi Uchiya, ${ }^{4}$ \\ Toshiaki Nikai ${ }^{4}$ and Kenji Ogawa ${ }^{1,2}$
}

\author{
${ }^{1}$ Department of Clinical Research, National Hospital Organization, Higashinagoya National Hospital, \\ 5-101 Umemorizaka, Meito-ku, Nagoya, Aichi 465-8620, Japan \\ ${ }^{2}$ Department of Pulmonary Medicine, National Hospital Organization, Higashinagoya National \\ Hospital, 5-101 Umemorizaka, Meito-ku, Nagoya, Aichi 465-8620, Japan \\ ${ }^{3}$ Department of Infectious Diseases, Center of National University Hospital for Infection Control, \\ Nagoya University Hospital, 65 Tsurumai, Showa-ku, Nagoya, Aichi 464-8560, Japan \\ ${ }^{4}$ Department of Microbiology, Faculty of Pharmacy, Meijo University, 150 Yagotoyama, \\ Tempaku-ku, Nagoya, Aichi 468-8503, Japan \\ ${ }^{5}$ Department of Pharmacy, National Hospital Organization, Nagoya Medical Center, 4-1-1 \\ Sannomaru, Naka-ku, Nagoya 460-0001, Japan
}

\begin{abstract}
In addition to its known status as a disseminated disease in HIV-positive patients, Mycobacterium avium complex (MAC) is increasingly recognized as a causative pathogen of respiratory disease in HIV-negative patients. MAC is divided into Mycobacterium avium, and the less-epidemiologically studied Mycobacterium intracellulare. Genetic typing for $M$. intracellulare using variable number of tandem repeats (VNTR) has not yet been developed. The aim of this study was to identify VNTR loci in the genome of $M$. intracellulare and apply them as an epidemiological tool to clinical isolates. Here, we identified 25 VNTR loci on the M. intracellulare genome, of which 16 showed variations among clinical isolates in the number of tandem repeat motifs. Among the $74 \mathrm{M}$. intracellulare isolates, 50 genotypes were distinguished using the 16 VNTR loci, resulting in a Hunter Gaston's discriminatory index of 0.988 . Moreover, all 16 VNTR loci were stable in different sets of isolates recovered within time intervals ranging from 2 to 1551 days from 14 separate patients. These results indicate that for use as epidemiological markers of $M$. intracellulare, the loci in this VNTR assay are highly discriminating and stable over time.
\end{abstract}

\section{INTRODUCTION}

Mycobacterium avium complex (MAC) is an opportunistic pathogen that causes human disease, and has been isolated from a variety of animal hosts and environmental sources such as soil, water and dust (Bauer et al., 1999; Dvorska et al., 2002; De Groote et al., 2006). MAC is divided into

Abbreviations: MAC, Mycobacterium avium complex; MLVA, multilocus variable number of tandem repeats analysis; PRA, PCR-restriction enzyme analysis; TR, tandem repeat; VNTR, variable number of tandem repeats.

The DDBJ accession number for the sequence reported in this paper is AB519649.

Three supplementary figures are available with the online version of this paper. two closely related organisms, Mycobacterium avium and Mycobacterium intracellulare (Inderlied et al., 1993), which together account for more than $70 \%$ of non-tuberculous mycobacterial disease in Japan. M. intracellulare is more frequently isolated from immunocompetent patients with pulmonary disease than from immunocompromised patients (Kyriakopoulos et al., 1997). Furthermore, immunocompetent patients with MAC disease can be either chronically infected with a given strain and relapse after treatment due to reactivation, or be reinfected by a different strain after cure. Although direct infection from human-to-human contact is considered unlikely (Wolinsky, 1979), and several studies have implicated drinking water or bathrooms as sources of MAC infection (von Reyn et al., 1994; Le Dantec et al., 2002; Nishiuchi 
et al., 2007), the source of infection in humans has not yet been clearly identified. The establishment of an accurate and reliable technique for the epidemiological detection and typing of MAC strains is therefore of considerable clinical importance.

PCR-based techniques for the identification and typing of MAC strains have been previously described. Telenti et al. (1993) reported PCR amplification of the gene encoding a $65 \mathrm{kDa}$ heat-shock protein ( $h s p 65)$, followed by PCRrestriction enzyme analysis (PRA) to determine mycobacterial species, including $M$. avium and M. intracellulare, although only four genotypes of $M$. intracellulare have been distinguished using this technique (Prammananan et al., 2006).

On the other hand, multilocus variable number of tandem repeats (VNTR) analysis (MLVA) has proven to be a rapid and highly discriminating subtyping method for several mycobacterial species (Stragier et al., 2005; Supply et al., 2006). Moreover, in recent studies, Bull et al. (2003), Thibault et al. (2007) and Inagaki et al. (2009) identified loci containing VNTR of specific mycobacterial interspersed repetitive units (MIRUs) in M. avium. However, no VNTR loci in $M$. intracellulare have yet been identified.

The aim of this study was to identify VNTR loci based on an intensive screening of tandem repeat (TR) loci in the $M$. intracellulare genome and to examine their epidemiological usefulness when applied to clinical isolates of $M$. intracellulare recovered from different patients.

\section{METHODS}

Bacterial strains. Reference strains (Mycobacterium intracellulare JATA52-01, derived from $M$. intracellulare ATCC 13950) were purchased from the Japan Anti-Tuberculosis Association. Ninetythree clinical strains, recovered from $74 \mathrm{HIV}$-negative patients with pulmonary disease, were identified as $M$. intracellulare using the Cobas Amplicor Mycobacterium Test (Roche Diagnostic Systems).

Preparation of genomic DNA. Clinical isolates of $M$. intracellulare were cultured at $37{ }^{\circ} \mathrm{C}$ for $1-3$ weeks in $7 \mathrm{ml}$ Middlebrook $7 \mathrm{H} 9$ liquid medium supplemented with $10 \%$ oleic acid albumin-glucose-catalase enrichment, and $200 \mu \mathrm{l}$ of this $7 \mathrm{ml}$ was cultured for enrichment in $5 \mathrm{ml}$ Mycobroth liquid medium (Kyokuto) to an $\mathrm{OD}_{530}$ of 0.2. DNA was then extracted using InstaGene Matrix (Bio-Rad) according to the manufacturer's instructions.

hsp65-PCR-restriction enzyme analysis (hsp65-PRA). A 439 bp fragment of the $h s p 65$ gene was amplified according to the procedure described by Telenti et al. (1993), using primers Tb11 (5'-ACCAACGATGGTGTGTCCAT-3') and Tb12 (5'-CTTGTCGAACCGCATACCCT- $3^{\prime}$ ), and then digested with BstEII or HaeIII (Takara Bio). After digestion, $12 \mu \mathrm{l}$ of the restriction digest was loaded onto a $4 \%$ agarose gel (E-gel; Invitrogen) and the gel was electrophoresed by the E-gel power system (Invitrogen). A 50 bp DNA Ladder (Invitrogen) served as an external molecular size marker. Fragments were visualized by ethidium bromide fluorescence, and lengths were calculated by Quantity One (Bio-Rad).

Selection of VNTR loci. The unfinished genome sequence of $M$. intracellulare ATCC 13950 (GenBank accession number
ABIN01000000), which was obtained from the NCBI database (http://www.ncbi.nlm.nih.gov/genomes/lproks.cgi) and is presently being sequenced at McGill University, was screened for TRs using the Tandem Repeat Finder (http://tandem.bu.edu). Among the TRs found, several different loci were selected using the criteria of: (i) minimum repeat size of $50 \mathrm{bp}$, allowing differentiation of the polymorphic VNTR loci by size on agarose gels, and (ii) $>90 \%$ conservation between the TRs. The VNTR loci used and their characteristics are listed in Table 1. Primers were designed utilizing Primer 3 software (http://frodo.wi.mit.edu/), and each primer was designed to anneal to the flanking regions of the TR. To confirm that each VNTR locus was different, VNTR sequences were compared with genome sequence data of M. intracellulare ATCC 13950 using BLASTN software.

Final selection of VNTR loci was based on the following criteria of Monteil et al. (2007): (i) candidate loci with less than two different alleles were excluded, (ii) when two VNTR loci candidates were isomorphic, one was excluded, and (iii) the full band patterns had to be readable.

VNTR analysis. Mixtures were prepared containing template DNA $(1 \mu \mathrm{l})$, dNTP mixture $(2.5 \mu \mathrm{l}), 10 \times$ PCR buffer $(2.5 \mu \mathrm{l})$, each of the primer sets (both $12.5 \mu \mathrm{M}$ ), $1.25 \mathrm{U}$ AmpliTaqGold (Applied Biosystems) and DMSO (Wako) to a final concentration of $4 \%$. Sterilized purified water was added, bringing the total volumes to $25 \mu \mathrm{l}$, and the mixtures were then subjected to PCR.

The PCR programme used consisted of an initial denaturing step at $95{ }^{\circ} \mathrm{C}$ for $10 \mathrm{~min}$, followed by 38 cycles of denaturing at $94{ }^{\circ} \mathrm{C}$ for $30 \mathrm{~s}$, annealing at $60{ }^{\circ} \mathrm{C}$ for $30 \mathrm{~s}$, extension at $72{ }^{\circ} \mathrm{C}$ for $60 \mathrm{~s}$, and a final extension step at $72{ }^{\circ} \mathrm{C}$ for $7 \mathrm{~min}$. The amplified DNA products were subjected to electrophoresis on $2 \%$ E-gel (Invitrogen) to determine their size in bp. Template DNA from $M$. intracellulare ATCC 13950 was used as a control in each experiment.

The estimated size of PCR products for the different alleles was deduced from the allele size range, and from the basic unit length. For units that remained incomplete, the calculated values were rounded down to the closest whole number; for example in VNTR-7 loci, $(53 \mathrm{bp} \times 3)+38 \mathrm{bp}$ units were considered as 3 repeat units (Fig. 1).

The allelic diversity of each VNTR locus was calculated using Selander's formula (Selander et al., 1986) and the discriminatory power of MLVA using Hunter and Gaston's formula (Hunter \& Gaston, 1988).

The PCR products obtained from various VNTR loci of $M$. intracellulare and clinical strains were purified using a GenElute PCR DNA Purification kit (Sigma-Aldrich), and direct sequencing analysis was performed with the same primers as used in the PCRs. Alignment of the nucleotide sequences was performed using the CLC Sequence Viewer 4.6.2 (CLC Bio).

Phylogenetic analysis. Phylogenetic analysis was estimated from Manhattan distance matrix data of the allele profiles of VNTR by Fitch-Margoliash criterion without an evolutionary clock using PHYLIP version 3.68c (Nishimori et al., 2002).

\section{RESULTS}

\section{Strain identification}

All 93 isolates recovered from sputum were identified as $M$. intracellulare using the Cobas Amplicor Mycobacterium Test, and determined as M. intracellulare hsp65 allelic variant I using hsp65-PRA. Although these results were 
Table 1. Primer sequences, tandem repeat motifs, conservation, size, and copy number of 16 VNTR loci in M. intracellulare ATCC 13950

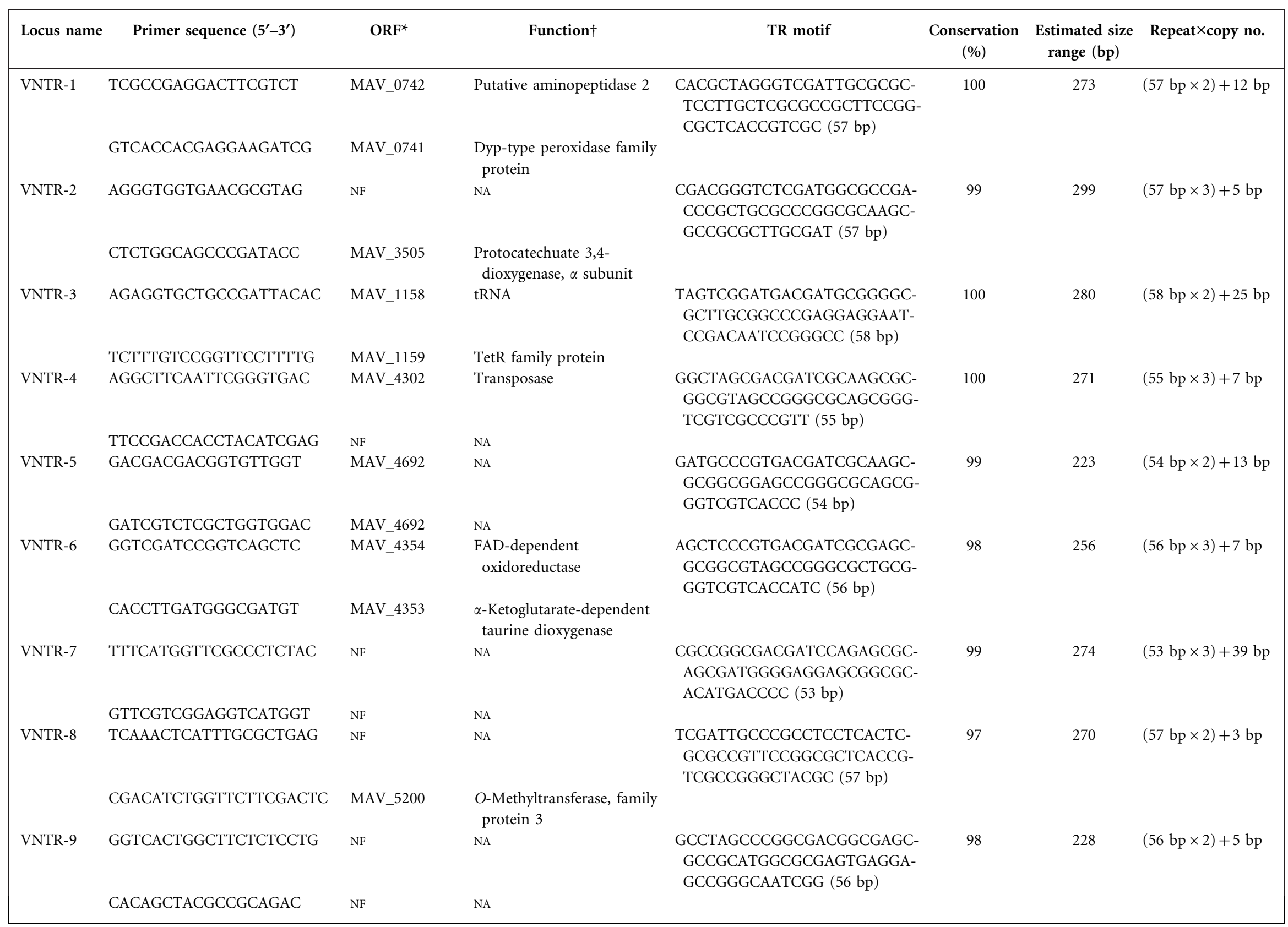


Table 1. cont.

\begin{tabular}{|c|c|c|c|c|c|c|c|}
\hline Locus name & Primer sequence $\left(5^{\prime}-3^{\prime}\right)$ & ORF $^{*}$ & Function $\dagger$ & TR motif & $\begin{array}{c}\text { Conservation } \\
(\%)\end{array}$ & $\begin{array}{l}\text { Estimated size } \\
\text { range (bp) }\end{array}$ & Repeat $\times$ copy no. \\
\hline \multirow[t]{2}{*}{ VNTR-10 } & GGCTGGTTCTTTCTGGTGAC & MAV_0237 & $\begin{array}{l}\text { Glycosyltransferase, group } \\
2 \text { family protein }\end{array}$ & $\begin{array}{l}\text { CCTAGTGGCGATCGCGAGCGCG- } \\
\text { GCGGAGCCGGGCGAAGCGGGT- } \\
\text { CGCCACCATCGATA (57 bp) }\end{array}$ & 98 & 353 & $(57 \mathrm{bp} \times 4)$ \\
\hline & CGCGTCAAGGAACGTCAT & MAV_0236 & $\begin{array}{l}\text { O-antigen export system, } \\
\text { permease protein }\end{array}$ & & & & \\
\hline \multirow[t]{2}{*}{ VNTR-11 } & TGTTGCGCTGAGGTCATATC & $\mathrm{NF}$ & NA & $\begin{array}{l}\text { AGCCCGGCGACGGCGAGCGCCG- } \\
\text { AAACGGCGCGAGTGAGGAGTC- } \\
\text { GGGCAATCCAACCA (57 bp) }\end{array}$ & 96 & 189 & $(57 \mathrm{bp} \times 2)+6 \mathrm{bp}$ \\
\hline & ACAGGTTGTCGGTCATTGGT & $\mathrm{NF}$ & NA & & & & \\
\hline \multirow[t]{2}{*}{ VNTR-12 } & AGACCAACCCAGAAAAGTGC & $\mathrm{NF}$ & NA & $\begin{array}{l}\text { ATGACTGCGACAGCGACGAAGC- } \\
\text { AGAGCGCAGCGATGAAGAGGA- } \\
\text { GTGGCGCTCG (53 bp) }\end{array}$ & 97 & 245 & $(53 \mathrm{bp} \times 2)+6 b p$ \\
\hline & GTCGTGATACGCCGAATTG & MAV_4451 & 30 S ribosomal protein $S 8$ & & & & \\
\hline \multirow[t]{2}{*}{ VNTR-13 } & GTTCAGCGAGCCGGTATCT & MAV_1628 & $\begin{array}{l}\text { Putative acyl-CoA } \\
\text { dehydrogenase }\end{array}$ & $\begin{array}{l}\text { TGAGGAGGAGCGGCGAAGGTGA- } \\
\text { CCGCCGGCGACGATGCAGAGC- } \\
\text { GAAGCGA (50 bp) }\end{array}$ & 97 & 292 & $(50 b p \times 3)+21 b p$ \\
\hline & AGCTCTCGCAGCTTGGTTC & MAV_1627 & Feruloyl-CoA synthetase & & & & \\
\hline \multirow[t]{2}{*}{ VNTR-14 } & ATGCCGGTTAGTCTCTCACG & MAV_4662 & $\begin{array}{l}\text { 2,3-Bisphosphoglycerate- } \\
\text { dependent phosphoglycerate } \\
\text { mutase }\end{array}$ & $\begin{array}{l}\text { GCATCGTCGTCTTCGCGGCTCA- } \\
\text { AAGCTCGACTCCTCCTCATCGC- } \\
\text { GCTTCGCGCTCT (56 bp) }\end{array}$ & 97 & 258 & $(56 \mathrm{bp} \times 2)+15 b p$ \\
\hline & GCTCGTCGATCCAGAAAGAG & MAV_4664 & $\mathrm{NA}$ & & & & \\
\hline \multirow[t]{2}{*}{ VNTR-15 } & GCAAACGCAGTGGTACTCAG & MAV_2430 & $\begin{array}{l}\text { Cobalamin biosynthesis } \\
\text { protein CobIJ }\end{array}$ & $\begin{array}{l}\text { CGTAGGGCTCAGTCTGATTGCC- } \\
\text { CTACTCCTCCGCACCCCGCCAC- } \\
\text { GCGGGCTGCATCGT (58 bp) }\end{array}$ & 93 & 219 & $(58 \mathrm{bp} \times 2)+13 b p$ \\
\hline & GATGATGCCGAGCACCTG & MAV_2429 & PPE family protein & & & & \\
\hline \multirow[t]{2}{*}{ VNTR-16 } & GGACGCTTTGTATCCGAGTT & MAV_4761 & $\begin{array}{l}\text { F420-dependent glucose-6- } \\
\text { phosphate dehydrogenase }\end{array}$ & $\begin{array}{l}\text { CTCCTGTGCTGATGGTCGCGAC- } \\
\text { CCGCCGCGCCCGGCTACGCCG- } \\
\text { CGCTTGCGATCG (55 bp) }\end{array}$ & 96 & 265 & $(55 \mathrm{bp} \times 2)+43 \mathrm{bp}$ \\
\hline & ACCGTGTCGGTGACTTGAAC & MAV_4762 & $\begin{array}{l}\text { Metallo- } \beta \text {-lactamase } \\
\text { superfamily protein }\end{array}$ & & & & \\
\hline
\end{tabular}

${ }^{\star} \mathrm{ORF}$, homologus open reading frame in M. avium 104 genome. NF, Not found.

$\dagger$ As predicted from M. avium 104. NA, Not applicable. 


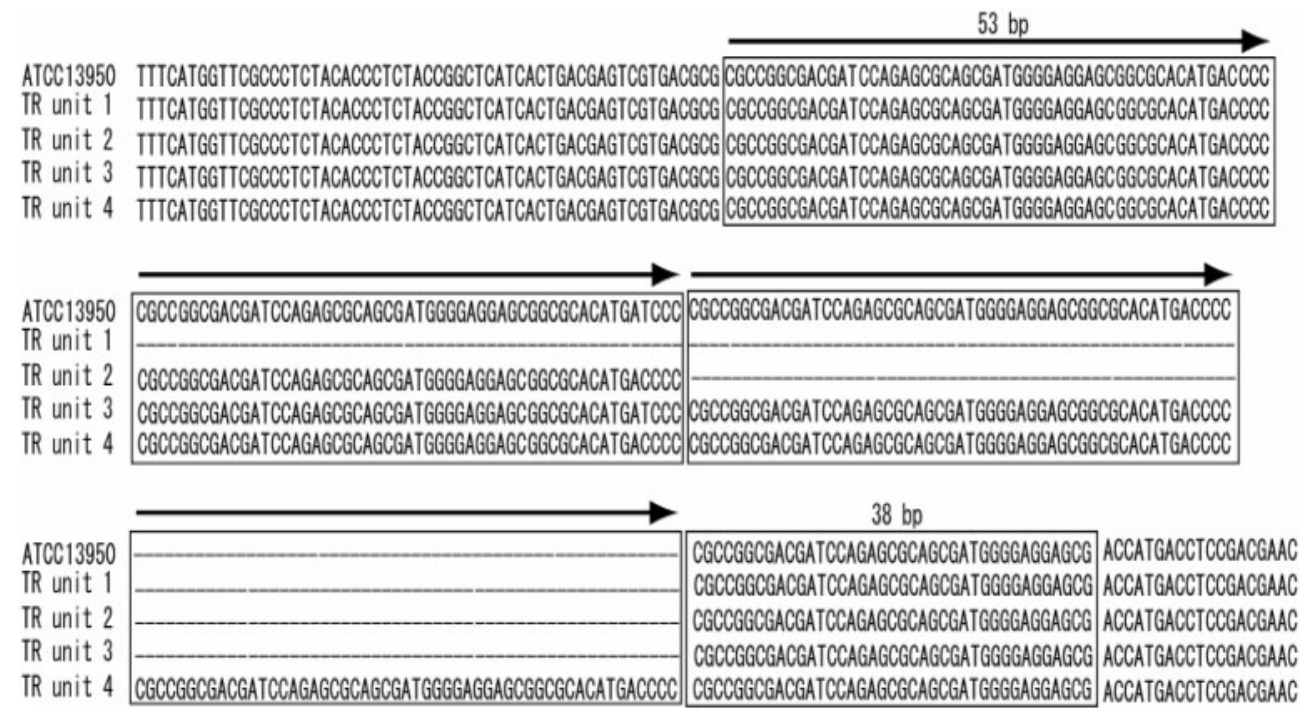

Fig. 1. Alignment of the M. intracellulare ATCC 13950 sequence with sequenced PCR product for clinical strains. The 53 bp repeat units are indicated by arrows. Partial repeats of varying length are present at the end of all the loci; the VNTR-7 locus contains three complete copies of the 53 bp repeat, and also an additional 38 bases corresponding to the beginning of another tandem repeat in $M$. intracellulare ATCC 13950.

consistent with a previous report from Thailand that showed $M$. intracellulare hsp 65 allelic variant I was the most predominant type among pulmonary specimen isolates (Prammananan et al., 2006), there were no PRA profiles that matched the described patterns of non-M. avium or non-M. intracellulare.

\section{In silico identification and selection of VNTR loci}

At the time of this study, only one M. intracellulare genome sequence was available online, and using this, 25 loci were initially chosen after comparing the lengths of repeats (repeats of $50-70 \mathrm{bp}$ ), sequence identities ( $>90 \%$ nucleotide sequence identity between repeats) and copy numbers (between two and five copies of the unit repeat). We focused on TRs of the mini-satellite class, defined by a repeat unit size in the range of $10-100 \mathrm{bp}$, as the corresponding allelic differences of these TRs can be easily resolved by agarose gel electrophoresis.

PCRs, however, gave no amplification or gave amplification of bands associated with TRs in several loci that were excluded from this study (data not shown). Moreover, we excluded the loci where an insertion sequence (ISMin1; DDBJ accession no. AB519649) was inserted downstream of the gene (see Supplementary Fig. S1, available with the online version of this paper).

Accordingly, we selected 16 loci based on the three criteria described above; their characteristics, together with the primers used for PCR amplification, are given in Table 1. The gel profile and sequencing data for 16 VNTR loci are indicated in Supplementary Figs S2 and S3. When they were applied to MLVA in the $93 \mathrm{M}$. intracellulare clinical isolates, and after the exclusion of identical profiles of isolates from the same patients, 74 representative allelic profiles were identified.

\section{Allelic diversity and distribution of VNTR allele numbers}

Table 2 shows the allelic diversity of various VNTR loci. The allelic diversity index $(h)$ was used to evaluate allelic diversity of the various VNTR loci. Based on this study, nine VNTR loci (VNTR-2, 4, 6, 7, 8, 10,11, 13 and 15) achieved a high diversity index $(h>0.5)$. Five VNTR loci (VNTR-1, 3, 5, 12 and 14) had a medium diversity index $(h<0.5)$, and VNTR-9 and 2 were the least discriminated loci $(h<0.1)$.

The discriminatory index of MLVA typing was calculated according to Hunter and Gaston's formula (Hunter \& Gaston, 1988), in which the closer the discriminatory index is to 1 , the higher the discriminatory power of the method. The MLVA method identified 50 distinct genotypes, consisting of 17 clustered and 33 unique, from 74 representative isolates (Fig. 2), giving a Hunter Gaston's Discriminatory Index for MLVA of 0.988 .

\section{Stability of the individual VNTR loci}

The VNTR profile varied considerably among strains. We therefore explored the frequency of mobility in individual VNTR loci by examining changes in the VNTR pattern during repeated passages in vitro and between serial in vivo isolates recovered from 14 separate patients. 
Table 2. VNTR allelic distribution in $M$. intracellulare clinical isolates

\begin{tabular}{|c|c|c|c|c|c|c|c|c|}
\hline \multirow{2}{*}{$\begin{array}{l}\text { Isolate and } \\
\text { locus }\end{array}$} & \multicolumn{7}{|c|}{ No. of isolates with the specified TR allele } & \multirow{2}{*}{$\begin{array}{c}\text { Allelic } \\
\text { diversity }\end{array}$} \\
\hline & 0 & 1 & 2 & 3 & 4 & 5 & 6 & \\
\hline VNTR-2 & 3 & 43 & 18 & 8 & 2 & 0 & 0 & 0.58 \\
\hline VNTR-3 & 7 & 62 & 5 & 0 & 0 & 0 & 0 & 0.27 \\
\hline VNTR-4 & 0 & 32 & 9 & 13 & 20 & 0 & 0 & 0.69 \\
\hline VNTR-6 & 0 & 20 & 35 & 16 & 3 & 0 & 0 & 0.65 \\
\hline VNTR-7 & 0 & 20 & 38 & 15 & 1 & 0 & 0 & 0.62 \\
\hline VNTR-8 & 35 & 0 & 37 & 2 & 0 & 0 & 0 & 0.52 \\
\hline VNTR-9 & 2 & 1 & 71 & 0 & 0 & 0 & 0 & 0.07 \\
\hline VNTR-10 & 1 & 44 & 3 & 1 & 20 & 5 & 0 & 0.56 \\
\hline VNTR-11 & 0 & 1 & 34 & 0 & 36 & 2 & 1 & 0.54 \\
\hline VNTR-16 & 0 & 4 & 70 & 0 & 0 & 0 & 0 & 0.09 \\
\hline
\end{tabular}

In order to assess the effect of in vitro passage, two $M$. intracellulare clinical strains were streaked on 7H11 agar plates and passaged 20 times over 180 days, with a single colony used for inoculation at each passage. After the passages, genomic DNA was extracted and these samples were subjected to VNTR analysis, in which the original cells and the passaged cells showed identical patterns. Furthermore, 14 sets of isolates that were recovered from the same patients at time intervals ranging from 2 to 1551 days were subjected to MLVA and showed identical VNTR profiles, again indicating the stability of the VNTR profiles (Table 3 ).

\section{DISCUSSION}

VNTRs of the mini-satellite class are valuable markers for genotyping several mycobacterial species, including M. avium. To our knowledge, this study is the first to perform an exhaustive screening of potential VNTR loci in this genome. This screening was based on in silico identification of TRs in the preliminary $M$. intracellulare genome and experimental testing of the polymorphism of the most interesting TR candidates using clinical $M$. intracellulare isolates.

This MLVA exhibited a diversity index value of 0.988 , meaning that this method is highly informative, and hence possesses great discriminatory power for identification of genetically similar strains. Although these results were based on a limited number of samples and should therefore be considered preliminary, this MLVA showed a high discriminating power for the genetic diversity found in $M$. intracellulare strains.

With regard to other typing methods, the standard PFGE and restriction fragment length polymorphism methods are labour-intensive, take approximately 1 week to complete and require a large amount of DNA. In contrast, MLVA typing is a simple and highly reproducible procedure that can be performed with very little DNA and regardless of whether the bacteria are living or dead. It can be performed on mycobacteria killed by heat or alcohol, therefore reducing biohazards. PCR analysis and electrophoresis can be completed in just one day. Results are digital from the outset, simplifying the comparison of large numbers of strains.

In this study, we analysed the genotype stability of MLVA in $M$. intracellulare by using serial isolates from patients infected over long periods (up to 4 years). The 16 VNTR loci were identical within groups of serial isolates in all 14 cases. As these VNTR loci appear to be highly stable over a period as long as 4 years, they all appear suitable for reliable follow-up of patients chronically infected with $M$. intracellulare.

Wallace et al. (1998) reported that isolates recovered after apparently successful drug therapy should be genotyped to distinguish between treatment failure and reinfection with another strain. The detection of changes in MLVA should therefore be useful in distinguishing between endogenous reactivation or exogenous reinfection.

Griffith et al. (2006) and Prammananan et al. (2006) performed PRA of a $441 \mathrm{bp}$ fragment of the hsp65 gene in $M$. intracellulare isolates from patients with chronic pulmonary disease. Griffith et al. (2006) found that all 47 M. intracellulare isolates belonged to $M$. intracellulare I, while Prammananan et al. (2006) found that $85 \%$ of pulmonary specimen isolates were $M$. intracellulare I. The hsp65-PRA type that was present in all strains of $M$. intracellulare I in our study was the same as that in these 


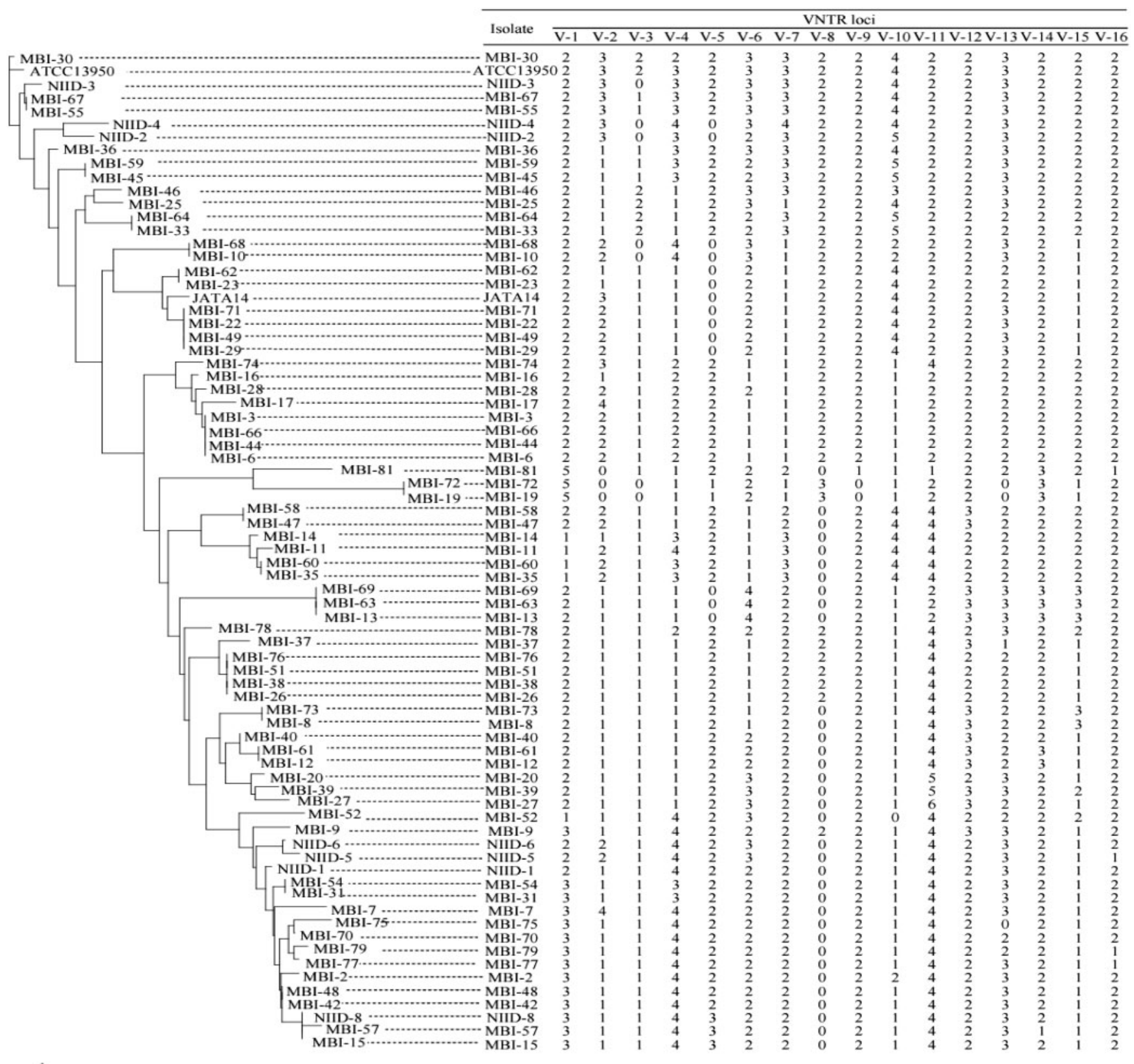

1

Fig. 2. MLVA dendrogram based on the allelic profiles of $74 \mathrm{M}$. intracellulare clinical isolates and $M$. intracellulare ATCC 13950. The Manhattan distance is indicated at the bottom.

studies (Griffith et al., 2006; Prammananan et al., 2006). Our study and these studies (Griffith et al., 2006; Prammananan et al., 2006) indicate that $M$. intracellulare I may possess particular pathogenic characteristics favourable for chronically infecting the pulmonary system. The fact that all clinical strains were identified as $h s p 65$ allelic variant I suggests, however, that hsp65PRA may not have sufficient discriminatory power for the genetic variants found in $M$. intracellulare clinical strains.
In conclusion, this MLVA method based on VNTR polymorphism provided a rapid and highly discriminating assay for the genetic typing of $M$. intracellulare. In addition, because of the objectivity of the output, the data are amenable to automated computer analysis for easier comparison across laboratories, indicating that MLVA typing may be widely accessible to research and public health laboratories. Elucidation of the transmission routes and pathophysiology of $M$. intracellulare infection may consequently advance through the use of MLVA typing. 
Table 3. Stability of VNTR genotypes in serial isolates from 14 chronically infected patients

The following strains were stable over the indicated time frames.

\begin{tabular}{|c|c|c|c|c|c|c|c|c|c|c|c|c|c|c|c|c|c|c|}
\hline $\begin{array}{l}\text { Patient } \\
\text { no. }\end{array}$ & $\begin{array}{l}\text { No. of } \\
\text { isolates } \\
\text { tested }\end{array}$ & $\begin{array}{c}\text { Time span } \\
\left(_{\text {days })^{\star}}\right.\end{array}$ & \multicolumn{16}{|c|}{ Copy no. for VNTR loci: } \\
\hline 1 & 4 & 1531 & 2 & 2 & 0 & 4 & 0 & 3 & 1 & 2 & 2 & 2 & 2 & 2 & 3 & 2 & 1 & 2 \\
\hline 2 & 5 & 973 & 2 & 1 & 1 & 3 & 2 & 2 & 3 & 2 & 2 & 5 & 2 & 2 & 3 & 2 & 2 & 2 \\
\hline 5 & 2 & 210 & 2 & 3 & 1 & 1 & 0 & 2 & 1 & 2 & 2 & 4 & 2 & 2 & 2 & 2 & 1 & 2 \\
\hline 6 & 2 & 184 & 1 & 2 & 1 & 3 & 2 & 1 & 2 & 0 & 2 & 4 & 4 & 2 & 2 & 2 & 2 & 2 \\
\hline 7 & 2 & 84 & 2 & 2 & 1 & 1 & 0 & 2 & 1 & 2 & 2 & 4 & 2 & 2 & 3 & 2 & 1 & 2 \\
\hline 8 & 2 & 39 & 2 & 1 & 1 & 2 & 2 & 1 & 1 & 2 & 2 & 1 & 4 & 2 & 2 & 2 & 2 & 2 \\
\hline 9 & 2 & 252 & 2 & 1 & 1 & 1 & 0 & 4 & 2 & 0 & 2 & 1 & 2 & 3 & 3 & 3 & 3 & 2 \\
\hline 10 & 2 & 35 & 2 & 1 & 1 & 1 & 2 & 3 & 2 & 0 & 2 & 1 & 5 & 3 & 3 & 2 & 3 & 2 \\
\hline 14 & 2 & 73 & 2 & 1 & 1 & 1 & 2 & 1 & 2 & 2 & 2 & 1 & 4 & 2 & 1 & 2 & 1 & 2 \\
\hline
\end{tabular}

*Time between the first and last isolates in each patient.

\section{ACKNOWLEDGEMENTS}

We thank Dr Keigo Shibayama (National Institute of Infectious Diseases) and Dr Satoshi Mitarai (Japan Anti-Tuberculosis Association) for providing $M$. intracellulare clinical isolates. Preliminary M. intracellulare ATCC 13950 genome sequence data were obtained from the NCBI database (http://www.ncbi.nlm.nih.gov/genomes/lproks.cgi). Sequencing of the $M$. intracellulare ATCC 13950 genome is currently under way at McGill University.

\section{REFERENCES}

Bauer, J., Andersen, A. B., Askgaard, D., Giese, S. B. \& Larsen, B. (1999). Typing of clinical Mycobacterium avium complex strains cultured during a 2-year period in Denmark by using IS1245. J Clin Microbiol 37, 600-605.

Bull, T. J., Sidi-Boumedine, K., McMinn, E. J., Stevenson, K., Pickup, R. \& Hermon-Taylor, J. (2003). Mycobacterial interspersed repetitive units (MIRU) differentiate Mycobacterium avium subspecies paratuberculosis from other species of the Mycobacterium avium complex. Mol Cell Probes 17, 157-164

De Groote, M. A., Pace, N. R., Fulton, K. \& Falkinham, J. O., III (2006). Relationships between Mycobacterium isolates from patients with pulmonary mycobacterial infection and potting soils. Appl Environ Microbiol 72, 7602-7606.

Dvorska, L., Bartos, M., Ostadal, O., Kaustova, J., Matlova, L. \& Pavlik, I. (2002). IS 1311 and IS 1245 restriction fragment length polymorphism analyses, serotypes, and drug susceptibilities of Mycobacterium avium complex isolates obtained from a human immunodeficiency virus-negative patient. J Clin Microbiol 40, 37123719.

Griffith, D. E., Brown-Elliott, B. A., Langsjoen, B., Zhang, Y., Pan, X., Girard, W., Nelson, K., Caccitolo, J., Alvarez, J. \& other authors (2006). Clinical and molecular analysis of macrolide resistance in
Mycobacterium avium complex lung disease. Am J Respir Crit Care Med 174, 928-934.

Hunter, P. R. \& Gaston, M. A. (1988). Numerical index of the discriminatory ability of typing systems: an application of Simpson's index of diversity. J Clin Microbiol 26, 2465-2466.

Inagaki, T., Nishimori, K., Yagi, T., Ichikawa, K., Moriyama, M., Nakagawa, T., Shibayama, T., Uchiya, K., Nikai, T. \& Ogawa, K. (2009). Comparison of a variable-number tandem-repeat (VNTR) method for typing Mycobacterium avium with mycobacterial interspersed repetitive-unit-VNTR and IS1245 restriction fragment length polymorphism typing. J Clin Microbiol 47, 2156-2164.

Inderlied, C. B., Kemper, C. A. \& Bermudez, L. E. (1993). The Mycobacterium avium complex. Clin Microbiol Rev 6, 266-310.

Kyriakopoulos, A. M., Tassios, P. T., Matsiota-Bernard, P., Marinis, E., Tsaousidou, S. \& Legakis, N. J. (1997). Characterization to species level of Mycobacterium avium complex strains from human immunodeficiency virus-positive and -negative patients. J Clin Microbiol 35, 3001-3003.

Le Dantec, C., Duguet, J.-P., Montiel, A., Dumoutier, N., Dubrou, S. \& Vincent, V. (2002). Occurrence of mycobacteria in water treatment lines and in water distribution systems. Appl Environ Microbiol 68, 5318-5325.

Monteil, M., Durand, B., Bouchouicha, R., Petit, E., Chomel, B., Arvand, M., Boulouis, H. J. \& Haddad, N. (2007). Development of discriminatory multiple-locus variable number tandem repeat analysis for Bartonella henselae. Microbiology 153, 1141-1148.

Nishimori, K., Uchida, I., Tanaka, K., Nishimori, T., Imai, K., Kashiwazaki, Y., Murata, N. \& Jinma, K. (2002). Molecular epidemiological manual for Mycobacterium tuberculosis complex and Mycobacterium avium using VNTR (Variable Numbers of Tandem Repeats) typing. Bull Natl Inst Anim Health 109, 25-32 (in Japanese).

Nishiuchi, Y., Maekura, R., Kitada, S., Tamaru, A., Taguri, T., Kira, Y., Hiraga, T., Hirotani, A., Yoshimura, K. \& other authors (2007). The recovery of Mycobacterium avium-intracellulare complex (MAC) from 
the residential bathrooms of patients with pulmonary MAC. Clin Infect Dis 45, 347-351.

Prammananan, T., Phunpruch, S., Tingtoy, N., Srimuang, S. \& Chaiprasert, A. (2006). Distribution of hsp65 PCR-restriction enzyme analysis patterns among Mycobacterium avium complex isolates in Thailand. J Clin Microbiol 44, 3819-3821.

Selander, R. K., Caugant, D. A., Ochman, H., Musser, J. M., Gilmour, M. N. \& Whittam, T. S. (1986). Methods of multilocus enzyme electrophoresis for bacterial population genetics and systematics. Appl Environ Microbiol 51, 873-884.

Stragier, P., Ablordey, A., Meyers, W. M. \& Portaels, F. (2005). Genotyping Mycobacterium ulcerans and Mycobacterium marinum by using mycobacterial interspersed repetitive units. J Bacteriol 187, 1639-1647.

Supply, P., Allix, C., Lesjean, S., Cardoso-Oelemann, M., RuschGerdes, S., Willery, E., Savine, E., de Haas, P., van Deutekom, H. \& other authors (2006). Proposal for standardization of optimized mycobacterial interspersed repetitive unit-variable-number tandem repeat typing of Mycobacterium tuberculosis. J Clin Microbiol 44, $4498-4510$.
Telenti, A., Marchesi, F., Balz, M., Bally, F., Böttger, E. C. \& Bodmer, T. (1993). Rapid identification of mycobacteria to the species level by polymerase chain reaction and restriction analysis. J Clin Microbiol 31, 175-178.

Thibault, V. C., Grayon, M., Boschiroli, M. L., Hubbans, C., Overduin, P., Stevenson, K., Gutierrez, M. C., Supply, P. \& Biet, F. (2007). New variable number tandem repeat markers for typing $M$. avium subsp. paratuberculosis and M. avium strains: comparison with IS900 RFLP and IS1245 RFLP typing. J Clin Microbiol 45, 2404-2410.

von Reyn, C. F., Marlow, J. N., Arbeit, R. D., Barber, T. W. \& Falkinham, J. O. (1994). Persistent colonisation of potable water as a source of Mycobacterium avium infection in AIDS. Lancet 343, 1137-1141.

Wallace, R. J., Jr, Zhang, Y., Brown, B. A., Dawson, D., Murphy, D. T., Wilson, R. \& Griffith, D. E. (1998). Polyclonal Mycobacterium avium complex infections in patients with nodular bronchiectasis. Am J Respir Crit Care Med 158, 1235-1244.

Wolinsky, E. (1979). Nontuberculous mycobacteria and associated diseases. Am Rev Respir Dis 119, 107-159.

Edited by: G. R. Stewart 